\section{BMJ Open Respiratory Research}

\title{
Using and implementing care bundles for patients with acute admission for COPD: qualitative study of healthcare professionals' experience in four hospitals in England
}

\author{
Ali Shaw, ${ }^{1}$ Katherine Morton, ${ }^{1}$ Anna King, ${ }^{1}$ Melanie Chalder, ${ }^{1}$ James Calvert, ${ }^{2}$ \\ Sue Jenkins, ${ }^{3}$ Sarah Purdy (1) ${ }^{1}$
}

To cite: Shaw A, Morton $\mathrm{K}$, King $A$, et al. Using and implementing care bundles for patients with acute admission for COPD: qualitative study of healthcare professionals' experience in four hospitals in England. BMJ Open Resp Res 2020;7:e000515. doi:10.1136/ bmjresp-2019-000515

Received 18 October 2019 Revised 23 January 2020 Accepted 23 January 2020
Check for updates

\section{(c) Author(s) (or their} employer(s)) 2020. Re-use permitted under CC BY-NC. No commercial re-use. See rights and permissions. Published by BMJ.

${ }^{1}$ Population Health Sciences, University of Bristol Faculty of Health Sciences, Bristol, UK

${ }^{2}$ Respiratory Medicine, North Bristol NHS Trust, Bristol, UK ${ }^{3}$ Independent Affiliated Consultant, University of Bristol, Bristol, UK

Correspondence to Professor Sarah Purdy; sarah.purdy@bristol.ac.uk

\section{ABSTRACT}

Background Care bundles are sets of evidence-based interventions to improve quality of hospital care at admission and discharge. Within a wider multi-method evaluation of care bundles for adults with an emergency admission for acute exacerbations of chronic obstructive pulmonary disease, a qualitative study was conducted. The aim was to evaluate how bundles were used, and healthcare professionals' experiences of the impact of bundles on the process of care delivery.

Methods Within the wider evaluation, four acute hospitals that were using COPD care bundles were purposefully sampled for geographical variation. Qualitative data were gathered through non-participant observation of patient care and interviews with healthcare professionals, patients and carers. This paper reports a thematic analysis of data from observation and interviews with professionals. Results Healthcare professionals generally experienced care bundles as positive for standardising working practices and patient care, valuing how bundles could support a clear care pathway for patients, enable transitions between settings and identify postdischarge support required by patients. Successful use of bundles was perceived as more likely with the presence of either (or both) a clinical champion for bundles and system-based initiatives such as financial incentives, within a local culture of quality improvement. Challenges in accurately diagnosing COPD hampered bundle use, including delivery of bundles to those subsequently considered ineligible, or missed opportunities to deliver admission bundles to those with COPD.

Conclusion Care bundles shape admission and discharge care processes for patients with COPD, from the perspective of staff involved in their delivery. However, different organisational, staff and clinical factors aid or hinder bundle use in an acute hospital context, suggesting potentially resolvable reasons for variable implementation of bundles. Finally, bundles may enhance staff experience of care delivery, even if the impact on patient outcomes remains uncertain.

\section{INTRODUCTION}

Chronic obstructive pulmonary disease (COPD) is a common respiratory disease,

\section{Key messages}

How are care bundles used for acute admissions for chronic obstructive pulmonary disease and what is the impact of bundles on delivery of care from the perspective of healthcare professionals?

- While there are barriers to successful implementation, bundles are perceived to enhance care delivery by standardising working practices and patient care, supporting a clear treatment pathway for patients, enabling communication between staff teams, and identifying postdischarge support needed by patients.

- It is vital to understand healthcare professionals' experiences of using quality improvement initiatives, as addressing the challenges to their use may enhance the process of care delivery even if the impact on patient outcomes remains uncertain.

with an estimated prevalence of over 3 million in UK of which only about 900000 have been diagnosed. ${ }^{1}$ COPD is a leading contributor to respiratory mortality, ${ }^{1}$ and accounts for $10 \%$ of hospital medical admissions in the UK (over 90000 annually), ${ }^{2}$ of whom $43 \%$ are readmitted within 90 days of discharge. ${ }^{3}$ COPD admissions have increased by $50 \%$ in the last decade, now accounting for one million bed days per year, ${ }^{4}$ and COPD care costs the National Health Service (NHS) between $£ 810$ million and $£ 930$ million annually. ${ }^{1}$ COPD requires reliable and sustainable solutions to effect change. ${ }^{5}$

There have been steps to improve COPD care, given the documented variation in treatment provision and outcomes across Europe and within the UK. ${ }^{36}$ Quality improvement initiatives include care bundles, which are sets of evidence-based interventions, elements of which are known to optimise 
Table 1 Summary of BTS admission and discharge bundles for AECOPD

BTS admission bundle

A correct diagnosis of AECOPD should be confirmed

An oxygen assessment should be undertaken and the correct target range prescribed within $30 \mathrm{~min}$

Recognise and respond to respiratory acidosis within 1 hour of admission

Medication (steroids and nebulisers) to be administered within 4 hours of admission

Review by respiratory team to take place within 24 hours of admission

\section{BTS discharge bundle}

All patients should have their respiratory medications and inhaler technique assessed prior to discharge.

All patients should receive a written plan for how to manage a further AECOPD and should receive a discharge pack of 'emergency' drugs prior to discharge.

Smoking status should be assessed together with a willingness to quit and for those patients indicating a wish for further assistance, a referral should be made to a stop smoking programme.

All patients should be assessed for their suitability for pulmonary rehabilitation prior to discharge.

Community follow-up within 2 weeks of discharge from hospital should be organised.

AECOPD, acute exacerbations of chronic obstructive pulmonary disease; BTS, British Thoracic Society.

clinical outcomes. ${ }^{2} 7$ The interventions are both necessary and sufficient; if any element is omitted, care will be less successful than if all elements are delivered. Bundles should be delivered collectively and consistently, focus on what care is delivered and how, should be easy to monitor and initiate quality improvement. In association with NHS improvement, the British Thoracic Society (BTS) developed admission and discharge care bundles for acute exacerbations of COPD (AECOPD). ${ }^{8}$ See table 1 for a summary of the care bundles.

Discharge bundles are used in various settings internationally. ${ }^{9}$ However, there is inconsistent evidence on the effectiveness and cost-effectiveness of admission and discharge bundles, or how their use impacts the process of care delivery.

We undertook a multi-method evaluation to address this knowledge gap. ${ }^{10-12}$ Our quantitative findings indicated that admission and discharge bundles for COPD had little impact on patient outcomes, including readmissions and mortality or healthcare costs, although bundles did appear to be associated with a reduced number of subsequent attendances at the emergency department. Variability in bundle structure and implementation create challenges in ascertaining the impact of COPD care bundles on patient outcomes. ${ }^{11} 12$ Within the evaluation, we conducted a qualitative study which examined how bundles were delivered and how staff perceived bundles to impact the process of patient care. ${ }^{1012}$ This paper develops a subset of these qualitative data relating to barriers and facilitators to implementation of bundles.

Lennox et al used documentary analysis of data recorded in quality improvement tools and focus groups with clinicians to identify barriers and facilitators to COPD care bundle implementation. ${ }^{13}$ They found that a key barrier was staffing issues (eg, staff shortages, lack of staff engagement and added workload of the bundle). Clinicians suggested facilitators to overcome these challenges including education to increase staff participation and payment frameworks to gain buy-in from managers. ${ }^{13}$ Going beyond this study, we observed actual use of COPD bundles and elicited the experiences of a range of staff involved in care bundle delivery in different acute hospital contexts. Our main evaluation paper noted that staff valued COPD care bundles for aiding care delivery. ${ }^{11}$ In this paper, we elaborate how the bundles were used in different hospital settings, how they impacted care delivery from the perspective of healthcare professionals and factors experienced to help or hinder successful bundle use. These qualitative findings may help to explain why bundles were not found to enhance patient outcomes, given the challenges to their implementation. ${ }^{1112}$

\section{METHODS}

We used a qualitative study design, incorporating nonparticipant observation and semi-structured interviews. In reporting our methods, we adhere to the Consolidated Criteria for Reporting Qualitative Research. ${ }^{14}$ The study was approved by the South West NHS Research Ethics Committee and research governance departments in each hospital.

\section{Sampling}

From 31 hospital sites participating in the wider evaluation, we purposefully sampled four hospitals across England that were using COPD care bundles, based on information from the BTS. ${ }^{10-12}$ These ranged from small, suburban to larger inner-city hospitals. All had an Emergency Department (ED), specialist respiratory ward(s) of varying sizes (30-70 beds) and specialist respiratory teams that included respiratory consultants, specialist respiratory nurses, physiotherapists and physiologists. Additional non-specialist staff involved in COPD care included junior doctors, general nursing staff, healthcare assistants and pharmacists. Three hospitals were delivering both admission and discharge bundles and one was delivering discharge bundles only.

Within each hospital we considered settings along the care pathway for patients with COPD including: the ED, 
Acute Medical Unit (AMU), respiratory ward, general wards and administrative areas such as the respiratory nursing office. For interviews with healthcare professionals, we sampled staff across a range of roles and backgrounds within these settings. We also interviewed community staff providing care for patients postdischarge to evaluate the impact on post-hospital management including self-care.

\section{Data collection}

Data were collected over 8 months (March to October 2016) by two experienced qualitative researchers (KM and $\mathrm{AK}$ ), using non-participant observation and interviews. Senior hospital staff were gatekeepers, providing access to the settings and individual participants. The researchers were not previously known to the research participants. Participants were not aware of the impact of care bundles on quantitative outcomes at the time of data collection.

Observation of delivery of admission and discharge bundles was conducted over a 2-week period in each hospital, Monday to Friday, between 07:00 and 19:0o. A flexible observation schedule was used and data were collected as written field notes, developed soon after the period of observation, replicated as a Word document and uploaded into NVivo. ${ }^{15}$

During the observation periods, face-to-face semistructured interviews were conducted with a range of hospital staff involved in delivering care bundles. Following each observation period, telephone interviews were conducted with community staff involved caring for patients with COPD postdischarge. Interviews followed a flexible topic guide and lasted between $20 \mathrm{~min}$ and 1 hour, depending on staff availability. Written consent was obtained prior to interview. All interviews were digitally recorded, fully transcribed, anonymised and uploaded into NVivo.

$\mathrm{KM}$ and AK met regularly with a senior qualitative researcher (AS) to review data collection plans and progress. Data were collected until the research team determined that the data had sufficient 'information power' to address the qualitative research aim. ${ }^{16}$

\section{Data analysis}

Following qualitative description of each hospital site, ${ }^{17}$ the observation and interview data across sites were examined using thematic analysis. ${ }^{18}$ A flexible coding framework was developed collaboratively and iteratively as data collection progressed. This included both deductive codes (based on the study aim) and inductive codes (derived from the data). The framework was refined into broader categories and higher-level themes, and data within themes were examined for disconfirming and confirming perspectives. Finally, an integrated account of each theme was written, illustrated by data from the observations and interviews, giving attention to the different perspectives represented. $\mathrm{KM}$ and $\mathrm{AK}$ wrote detailed descriptions of the sites and developed the

\begin{tabular}{lccc}
\hline Table 2 & Number of interviews in each hospital \\
\hline & $\begin{array}{l}\text { Community } \\
\text { Hospital staff } \\
\text { interviews }^{*}\end{array}$ & $\begin{array}{l}\text { Comf } \\
\text { staff } \\
\text { interviewst }\end{array}$ & Total \\
\hline IMP01 & 8 & 4 & 12 \\
IMP03 & 6 & 3 & 9 \\
IMP05 & 4 & 9 & 13 \\
IMP11 & 7 & 2 & 9 \\
\hline Total & 25 & 18 & 43 \\
\hline
\end{tabular}

*Including respiratory consultants, respiratory nurses, physiotherapists and junior doctors.

†Including community respiratory nurses, case load managers, community physiotherapists and general practitioners. GPs, general practitioners; IMP, implementation site.

coding framework. AS and SP independently conducted preliminary coding of a subset of the data, reviewed the coding framework and agreed the final themes, to enhance the trustworthiness of the analysis. KM and AS drafted the integrated accounts of themes, with input from AK and SP. The analysts had backgrounds in qualitative social science, admissions research and clinical medicine.

\section{RESULTS}

Across the four hospitals using care bundles, 106 hours of observation and 43 staff interviews were conducted. In the results, hospitals, settings and participants are identified by unique anonymous identifiers. Hospitals are identified as implementation site (IMP) and a number. Settings are identified by a descriptor (eg, MAU for Medical Admissions Unit) and a number. Staff participants are identified by role (eg, nurse). The healthcare professional interviews are summarised in table 2. The results are presented as a description of the context and process of bundle use derived from the observation and interviews, followed by two major themes (perceived impact of care bundles on care delivery; enablers and barriers to implementation of bundles) each with subthemes.

\section{Context and process of care bundle use}

Observation and interviews within each hospital revealed challenging local contexts within which healthcare professionals viewed themselves as 'firefighting'. This constrained their capacity to deliver quality care and take on quality improvement initiatives such as care bundles. Experiences of 'lacking resources', being 'understaffed' and a continually fluctuating population of staff inhibited their ability to treat patients with COPD as consistently and thoroughly as they would like. From the perspective of senior clinicians, local struggles were situated within a national context of NHS funding cuts and increasing pressure on primary and secondary care, compounded by an ageing population and increasing comorbidities. Clinicians felt that these factors combined to produce a 
growing population of older patients with multiple conditions, with 'a greater need for social support and care at home which is currently completely lacking', resulting in a growing number of hospital admissions.

Observations revealed variation in how care bundles were used. Roles and responsibilities for delivering admissions bundles differed across sites, although these were often allocated to respiratory nurses. The admission bundle was intended to be carried out in the ED, as per BTS guidelines. ${ }^{19}$ However in practice, it was predominantly delivered in the AMU, and often outside the specified 4 hours admission window. Admission bundles were generally delivered through completion of a paper form or sticker, with one site using an electronic form. Observations showed more consistent responsibilities and practices for delivery of the discharge bundle across hospitals. The respiratory nursing team led delivery, working with staff with other roles and varied grades to identify appropriate patients and deliver the bundle. Delivery was recorded by entering information onto paper forms at the patient's bedside, generally within a 24 hours window predischarge. Observation of delivery of discharge bundles indicated that the amount of time respiratory nurses spent with patients varied between 15 and $60 \mathrm{~min}$, depending on staff availability and patient need. At all four hospitals, attempts were made to follow-up patients within 24-48 hours post-discharge, to identify issues which might prompt readmission and to provide reassurance.

Working within these challenging local contexts, healthcare professionals had varying perceptions and experiences regarding the impact of bundles on care processes and the enablers and barriers to bundle implementation.

\section{Perceived impact of bundles on care delivery}

During interviews, staff expressed largely positive views of care bundles in terms of experienced impact on working practices and patient care. Across the four hospitals they noted that, in comparison to care without bundles, using bundles improved the respiratory team's ability to identify patients with COPD, including those admitted to non-respiratory wards. They agreed that bundles enabled more standardised and consistent care across settings.

Within this theme, three subthemes were identified relating to the perceived value of bundles for facilitating transitions of care between settings, enhancing the management of complex patients through systematic identification of patients' needs and prompting timely review by a respiratory specialist. See box 1 for illustrative quotes.

\section{Facilitating transitions between settings through enhanced communication}

Healthcare professionals valued care bundles for aiding transitions between settings, within hospitals or across acute and community services, through prompting information provision and communication between staff. Transitions within a hospital might include handover of patients between wards (eg, admission onto the respiratory ward from AMU), and transitions between secondary and primary care during and following discharge. At such points, poor communication could mean a lack of information about patients' treatment needs and delays in referrals. However, staff felt that bundles prompted effective communication and referral to appropriate services, such as pulmonary rehabilitation.

Across the four hospitals, staff highlighted the importance of support for patients with COPD on discharge in order to avoid readmission. An 'ineffective' discharge with poor community support could cause a patient to quickly 'bounce back' to the ED. Part of the purpose of discharge bundles was to avoid this, by referring patients to targeted

\section{Box 1 Illustrative quotes for perceived impact of bundles on care delivery}

\section{Facilitating transitions between settings through enhanced communication}

(there has been) a huge increase in the uptake of pulmonary rehab, I think it was something like a 30\%-70\% increase, just from implementing the discharge bundle. (Interview, IMP11 ACU1, Respiratory Nurse Specialist)

I think the communication coming back from hospital... is often lacking so it will often be a case of us manually having to go through the notes to see if they are back out of hospital again, or just a case of keeping an electronic list and then following them up. (Interview, IMP05 COM9, GP)

\section{Enhancing management of complex patients through systematic identification of patients' needs}

'It means that they [(patients]) get the care they need, every time, it's always standard, it's always how they should be and we know it's always been done' (Interview, IMP03, ACU6, Lead Nurse, Acute Care)

It's good because there are quite a few clinicians within our team, although we all review the patients, everyone is an individual and they may not necessarily focus in on the same aspects, so with the care bundle you know there is a flow chart, what needs to be covered, and you are ensuring that all patients received the same care, rather than on an ad hoc basis. (Interview, IMPO3 ACU4, COPD Nurse)

\section{Prompting timely specialist respiratory review}

with a care bundle, there is a better chance they are going to go out on the right treatment really, particularly if they have not been under the respiratory team, and they will have access to more services. (Interview, IMP11 ACU7, ED Consultant)

We always screen patients. Every morning as part of our team we screen patients that have come in, admitted with a COPD diagnosis. We then go down and see them in AMU. Every patient we identify we'll go down and review them. (Interview, IMP01 ACU1, Respiratory Nurse)

$\mathrm{ACU}$, acute care unit; COPD, chronic obstructive pulmonary disease; ED, Emergency Department; GP, general practitioner; IMP, implementation site. 
community services and by ensuring that community teams were aware that a patient was being discharged.

Community staff agreed with the importance of information exchange at discharge. Facilitated by discharge bundles, communication from the acute respiratory team to the community respiratory team could enable discharges to be flagged to primary care, and complexities regarding a patient's circumstances to be noted. General practitioners (GPs) noted that communication from the hospitals to the community was not always ideal, even when bundles were in place. One GP suggested that the discharge bundle might be improved by including an element that addressed communication with the primary care team. Routine follow-up telephone calls postdischarge, referral and monitoring by community nursing staff and patient education about inhalers and rescue packs were identified by GPs as options to help support patients in the community and avoid readmission.

\section{Enhancing management of complex patients through systematic identification of patients' needs}

A recurring issue for healthcare professionals across the four hospitals was the complexity of the COPD population, for example, comorbidities, social isolation and poor self-management behaviours. This complexity was seen to be a key motivator for quality improvement measures, including care bundles. Hospital respiratory teams felt that managing such a complex patient population requires them to have detailed knowledge of patients, and opportunities for detailed assessment of patients' treatment and follow-up care needs.

While healthcare professionals sought to develop this knowledge about each patient, they appreciated the role of bundles in enabling them to identify care pathways with an awareness of patient comorbidities, their living situations and challenges they might face in the community. Bundles provided enhanced opportunities to systematically identify and address the full range of patients' care needs, within hospital and once discharged to the community, and to standardise care.

From the perspective of hospital staff, use of an admission bundle allowed different staff with diverse roles within the respiratory team to consistently identify the needs of new patients as well as revisiting the needs of well-known frequent attenders, and to enable provision of care of a uniform standard.
Use of a discharge bundle was seen to be a helpful way to spend time identifying patients' self-management needs and pathways of care postdischarge. Staff valued the opportunity to prepare patients for discharge and make them aware of support services in the community, contributing towards prevention of readmission.

\section{Prompting timely specialist respiratory review}

Access to specialist respiratory review was viewed as key during admission and treatment of a patient with COPD in hospital. Staff identified timely specialist review as a crucial part of the admission care bundle that could impact significantly on patient outcomes and the likelihood of readmission. Specialist respiratory review prompted by a care bundle could ensure that the patient diagnosis was accurate, that chances of admission onto a respiratory ward (rather than a general ward) were increased, and that the patient would leave hospital with an appropriate treatment plan.

Healthcare professionals identified that within the broader context of high demand for hospital beds, it was not always possible for patients to be admitted onto a respiratory ward. Instead, the bundle prompted staff to screen the admission system for patients admitted with COPD, and staff would then locate these patients, either in AMU or on a ward, for a respiratory review. By providing respiratory review as close to admission as possible, the patient became known to the respiratory team and their care could be more effectively co-ordinated.

\section{Enablers and barriers to implementation of care bundles}

The second major theme concerns factors that enable or hinder implementation of care bundles from the perspective of healthcare professionals. Within this are two sub-themes: the local quality improvement culture and misdiagnosis of COPD. Box 2 provides illustrative quotes.

\section{Local quality improvement culture: resources, staffing, leadership and training}

From the perspective of healthcare professionals within the hospitals, a key factor that could enable but sometimes hinder bundle implementation was the local quality improvement culture, including resources, staffing, leadership and training. The interviews suggested

Box 2 Illustrative quotes for factors impacting implementation of care bundles

Quality improvement culture: resources, staffing, leadership and training 'some of those individuals were more easy to persuade once the CQUIN started because they then saw that if we didn't do this, we would have a chunk of money taken away from us at the end of the year... the CQUIN was to get much greater buy in at managerial level (IMP05 ACU4, Respiratory Consultant)

'we've gone from a zero per cent discharge bundle right because there were no staff to delivery it, to a 94\% delivery rate' (IMP05, ACU4, Respiratory Consultant

ACU, acute care unit; CQUIN, Commissioning for Quality and Innovation; IMP, implementation site. 
considerable variation in organisational culture across the hospitals, including: a senior 'champion' compared with a more system-led strategy; and staff-led, bottom-up approaches compared with top-down Trust or Clinical Commissioning Group-led strategies around organisational change.

According to the staff interviewed, two of the hospitals had historically used a Commissioning for Quality and Innovation (CQUIN) incentive in order to implement COPD care bundles. CQUINs were introduced in the NHS in 2009 and are financial incentives driven by targets, in this case for completion of admission or discharge bundles. Staff reported CQUINs to be successful drivers for compliance in bundle implementation, as the financial incentive obtained the necessary managerial 'buy in' and ring-fencing of resource.

Allocation of resources during the CQUIN was seen to have enabled hospitals to set-up systems (both electronic and paper) to record and monitor bundle completion, and for clinical staff to develop working relationships with trust analysts for monitoring bundle completion. Staff felt that such systems had the potential to be maintained beyond the end of the CQUIN and to support longer-term quality improvement, if appropriately resourced.

Designating staff responsibility for care bundles and resourcing such roles was perceived to be key for aiding bundle use. In hospitals with CQUINs, multi-disciplinary teams (MDTs) had been established to deliver care bundles and their ongoing presence was an indicator of success and longevity of implementation. MDTs were helpful for interdisciplinary communication about patients with COPD with complex needs, discussion of bundle completion rates, resolution of barriers to achieving targets and communication between acute and community teams. However, additional funding for such roles was often short-term, leaving questions about how effective implementation would be once these roles no longer existed.

While many healthcare professionals highlighted the positive impact of leadership by designated staff, there were also perceived dangers in this approach. Responsibility for bundle delivery could be too concentrated around one person rather than being a collective endeavour, even if there was buy-in from other staff. Having a single point of responsibility for bundle delivery was seen to be precarious and a major hurdle to successful wider implementation. Disruption to bundle use could also be introduced by conflict between staff members or competing priorities and demand for resources.

Staff also noted the need for education and training in quality improvement, including care bundles (components and monitoring) if change was to be embedded within the organisation. Such training could help to engage staff, show the value of bundles and provide guidance on successful implementation.
Misdiagnosis of COPD

From the perspective of senior staff associated with strategic aspects of bundle implementation, a key factor hindering the effective use of care bundles was the "misdiagnosis' of COPD, which they felt negatively impacted the extent to which they could appropriately use bundles and meet delivery targets. They perceived that misdiagnosis was a common problem. For example, one respiratory consultant estimated that at his hospital, '30\% of the diagnosis is wrong' in relation to COPD and he perceived that this misdiagnosis was particularly common in patients with asthma.

In the view of these healthcare professionals, misdiagnosis could 'artificially inflate' the population with COPD and add to the workload of the respiratory team. It could impact the appropriate use of care bundles by leading to unwarranted delivery of COPD care bundles to misdiagnosed patients, or cause failure to appropriately deliver a bundle to patients whose care might benefit from bundle use.

In contrast, healthcare professionals argued that accurate diagnosis of COPD could enable a patient to be treated on the correct care pathway, with appropriate application of care bundles, and referral to targeted community support and services postdischarge.

\section{DISCUSSION}

\section{Summary of results}

The aim of this qualitative study was to examine healthcare professionals' experiences and perceptions of care bundles for patients with COPD, within a wider multimethod evaluation. Overall, care bundles were experienced by staff as helpful for standardising working practices and patient care, supporting a clear care pathway for patients, enabling communication between staff teams and identifying postdischarge support needed by patients. In terms of context, greater perceived success of bundle implementation was associated with the presence of either (or both) a clinical champion for care bundles and system-based initiatives such as financial incentives, within a local culture of quality improvement. Difficulties in identifying which patients had a confirmed diagnosis of COPD at admission hampered bundle implementation, including delivery of bundles to those subsequently considered ineligible, or missed opportunities to deliver admission bundles to those with COPD who were "misdiagnosed'.

\section{Results in the context of relevant research}

Previous research has found that discharge bundles for COPD may help to reduce readmissions to hospital and are a means of embedding reliable, sustainable quality improvement with senior clinical support, training and monitoring. ${ }^{78}$ However, implementing discharge bundles without these supports has been shown to be challenging. ${ }^{13}$ Our wider evaluation concluded that given the low level of bundle uptake in study implementation 
sites, we do not yet know if COPD care bundles change patient care and outcomes when effectively implemented. ${ }^{11} 12$ The qualitative findings reported here shed light on possible reasons for poor implementation but also how bundles are valued by staff for enhancing their experiences of care delivery. Our findings identify challenges to the delivery of both bundles but indicate that admission bundles are more difficult to implement consistently, given the diversity of settings and clinical teams involved in care at admission.

Building on other work that has illustrated structural and resource threats to implementation, ${ }^{20}$ this study highlights the importance of local context and leadership, including clinical champions, ${ }^{21}$ staffing and resource factors in the delivery of a quality improvement initiative such as care bundles. Despite considerable investment by individual champions and health systems, our wider evaluation found that COPD bundles did not achieve the clinical outcomes delivered by an intensive pilot study among 'early adopters'. 81112 The findings of our qualitative study indicate why bundles may have had little impact on the measured quality of care, through highlighting local contextual challenges and barriers to bundle uptake from the perspective of staff involved in their delivery. These qualitative findings also support the value of understanding staff experiences of using quality improvement initiatives such as bundles, as from their perspective bundles may enhance care processes, even if the impact on patient outcomes remains uncertain.

\section{Implications}

Organisational pressures, the absence of a local quality improvement culture and misdiagnosis of COPD can hinder delivery of the high-quality evidence-based COPD care that health professionals desire to give. While we found evidence of more consistent delivery of the discharge bundle in four hospitals using bundles, the complexity of the admission process makes admission bundles more difficult to implement and monitor, which means implementation fell short of BTS targets for the delivery of key components of COPD care. ${ }^{19}$ To improve implementation, it may be beneficial to develop an implementation strategy that clarifies how bundles are expected to work and how they would enhance patient care. The strategy should account for factors impacting successful implementation identified in this study, and involve healthcare professionals in its development, as their engagement is key for ensuring an implementation strategy that is acceptable and workable for staff on the ground. The core components of the strategy would need to be delivered consistently, but with flexibility to allow adaptation to local context. ${ }^{22}$

\section{Strengths and limitations}

This qualitative study focuses on an important clinical issue that continues to pose a challenge for the NHS. We used a mixture of rigorous data collection methods, and analysis was conducted systematically by a multidisciplinary team with appropriate expertise in qualitative social science, respiratory medicine, admissions research and primary care.

Inclusion of service managers and commissioners may have offered more insight into strategic decision-making around quality improvement, and the governance and funding structures in which they are embedded. Observations at the weekend, overnight, over a longer time period or at other hospital sites could have produced alternative findings. Observation by other researchers, including those with a clinical background, may have produced data with a different focus. By collecting data between March and October, we may have missed the period of highest admissions given the seasonality to COPD exacerbations. How care bundles are used may also vary over time within hospitals; our data provide only a snapshot of these processes. Participating hospitals welcomed observation of their practice and it is possible that this selfselecting group felt they were performing well enough to survive scrutiny.

\section{CONCLUSION}

From the perspective of healthcare professionals working in acute contexts where care bundles are used, bundles can be valuable for enhancing the process of care delivery within hospitals, and across acute and primary care. Bundles have the potential to support quality improvement for patients with an emergency admission for COPD, with appropriate resourcing and collective buyin, within a local culture of quality improvement. There are barriers and facilitators to bundle use, including organisational challenges, which need to be addressed before they can be more successfully implemented. It is vital to understand staff experiences of using quality improvement initiatives, as addressing the challenges to their use may enhance the process of care delivery even if the impact on patient outcomes remains uncertain.

Acknowledgements This research has been conducted independently by the University of Bristol in partnership with staff from the University of the West of England, Bristol, North Bristol NHS Trust and the British Thoracic Society. We thank the patients, carers and healthcare professionals who contributed to the study, including our patient and public involvement panel. We are also grateful for the advice of our Study Steering Committee. The paper from our main evaluation was published in this Springer Journal in 2019 https://link.springer.com/content/pdf/10. 1186/s12890-016-0197-1.pdf

Contributors AS and SP designed the study. KM and AK collected and analysed the data, with support from AS and SP. AS, KM and SP drafted the paper. All authors read and approved the final manuscript.

Funding This work was supported by the National Institute for Health Research (NIHR) Health Services and Delivery Research (HS\&DR) Programme, project number $12 / 130 / 53$. The research was hosted by NHS Bristol and was designed and delivered in partnership with the Bristol Randomised Trials Collaboration (BRTC), a UK Clinical Research Collaboration (UKCRC) registered Clinical Trials Unit (CTU). The views expressed are those of the authors and not necessarily of the NHS, the NIHR or the Department of Health and Social Care.

Competing interests SP is a general practitioner, and JC is a hospital consultant working in the field of respiratory medicine. JC worked with colleagues at the British Thoracic Society to design and evaluate care bundles as an intervention to improve outcomes in different respiratory conditions, including chronic obstructive pulmonary disease, pneumonia and asthma. SP is a member of the National 
Institute for Health Research (NIHR) Health Services and Delivery Research Researcher-led Panel, from 2017 to date. SJ runs an independent consultancy for public and charitable sector clients, providing strategy and organisation development, leadership coaching and facilitation.

\section{Patient consent for publication Not required.}

Ethics approval Ethical approval was given by the South West (Frenchay) Research Ethics Committee (study reference 14/SW/1057). All participants consented to be interviewed and/or observed, and for their anonymised data to be published in study outputs.

Provenance and peer review Not commissioned; externally peer reviewed.

Data availability statement All data relevant to the study are included in the article or uploaded as supplementary information. Selected relevant data are presented in the article. We are unable to provide access to the original data as participants did not consent to open access to their data for future analysis.

Open access This is an open access article distributed in accordance with the Creative Commons Attribution Non Commercial (CC BY-NC 4.0) license, which permits others to distribute, remix, adapt, build upon this work non-commercially, and license their derivative works on different terms, provided the original work is properly cited, appropriate credit is given, any changes made indicated, and the use is non-commercial. See: http://creativecommons.org/licenses/by-nc/4.0/.

ORCID iD

Sarah Purdy http://orcid.org/0000-0002-3445-986X

\section{REFERENCES}

1 Department of Health. Consultation on a strategy for services for chronic obstructive for chronic obstructive pulmonary disease pulmonary disease (COPD) in England, 2010. Available: https://www. gov.uk/government/uploads/system/uploads/attachment_data/file/ 213840/dh_113279.pdf [Accessed 8 Oct 2019].

2 Stone RA, Lowe D, Holzhauer-Barrie J. COPD: who cares matters. National chronic obstructive pulmonary disease (COPD) audit programme: clinical audit of COPD exacerbations admitted to acute units in England and Wales 2014. London: Royal College of Physicians, 2015.

3 Price LC, Lowe D, Hosker HSR, et al. Uk national COPD audit 2003: impact of hospital resources and organisation of care on patient outcome following admission for acute COPD exacerbation. Thorax 2006;61:837-42.

4 British Lung Foundation. The battle for Breath- the impact of lung disease in the UK. London: British Lung Foundation, 2016.

5 lacobucci G. Hospital readmissions for COPD in England are rising, audit shows. BMJ 2017;356:j557.

6 Kayyali R, Odeh B, Frerichs I, et al. COPD care delivery pathways in five European Union countries: mapping and health care professionals' perceptions. Int J Chron Obstruct Pulmon Dis 2016;11:2831-8.

7 Resar R, Griffin FA, Haraden C, et al. Using care bundles to improve health care quality. IHI innovation series white paper. Cambridge, Massachusetts: Institute for Healthcare Improvement, 2012.

8 Turner AM, Lim WS, Rodrigo C, et al. A care-bundles approach to improving standard of care in AECOPD admissions: results of a national project. Thorax 2015;70:992-4.

9 Ospina MB, Mrklas K, Deuchar L, et al. A systematic review of the effectiveness of discharge care bundles for patients with COPD. Thorax 2017;72:31-9.

10 Chalder MJE, Wright CL, Morton KJP, et al. Study protocol for an evaluation of the effectiveness of 'care bundles' as a means of improving hospital care and reducing hospital readmission for patients with chronic obstructive pulmonary disease (COPD). BMC Pulm Med 2016;16:35.

11 Morton K, MacNeill S, Sanderson E, et al. Evaluation of 'care bundles' for patients with chronic obstructive pulmonary disease (COPD): a multisite study in the UK. BMJ Open Respir Res 2019;6:e000425.

12 Morton K, Sanderson E, Dixon P, et al. Care bundles to reduce re-admissions for patients with chronic obstructive pulmonary disease: a mixed-methods study. Health Serv Deliv Res 2019;7:1-128.

13 Lennox L, Green S, Howe C, et al. Identifying the challenges and facilitators of implementing a COPD care bundle. BMJ Open Respir Res 2014;1:e000035.

14 Tong A, Sainsbury P, Craig J. Consolidated criteria for reporting qualitative research (COREQ): a 32-item checklist for interviews and focus groups. Int J Qual Health Care 2007;19:349-57.

15 QSR International. NVivo qualitative data analysis software version 10, 2012.

16 Malterud K, Siersma VD, Guassora AD. Sample size in qualitative interview studies: guided by information power. Qual Health Res 2016;26:1753-60.

17 Sandelowski M. Whatever happened to qualitative description? Res Nurs Health 2000;23:334-40.

18 Braun V, Clarke V. Using thematic analysis in psychology. Qual Res Psychol 2006;3:77-101.

19 British Thoracic Society. Guidelines. Available: https://www.britthoracic.org.uk/standards-of-care/guidelines [Accessed 8 Oct 2019].

20 Dixon-Woods M, Tarrant C, Willars J, et al. How will it work? A qualitative study of strategic stakeholders' accounts of a patient safety initiative. Qual Saf Health Care 2010;19:74-8.

21 Miech EJ, Rattray NA, Flanagan ME, et al. Inside help: an integrative review of champions in healthcare-related implementation. SAGE Open Med 2018;6:205031211877326.

22 The Health Foundation. Evidence scan: improvement science, 2011. Available: https://www.health.org.uk/sites/default/files/ ImprovementScience.pdf [Accessed 8 Oct 2019]. 